 \\ Annales de Bretagne et des Pays de l'Ouest
}

Anjou. Maine. Poitou-Charente. Touraine

125-3 | 2018

Saint-Malo, construction d'un pôle marchand

(1500-1660)

\section{Au cœur des échanges européens : argent américain et circuits économiques entre la Bretagne et l'Espagne (1570-1635)}

At the heart of European exchanges: American money and economic circuits between Brittany and Spain (1570-1635)

Jean-Philippe Priotti

\section{OpenEdition}

Journals

\section{Édition électronique}

URL : http://journals.openedition.org/abpo/3958

DOI : $10.4000 / a b p o .3958$

ISBN : 978-2-7535-7691-9

ISSN : 2108-6443

\section{Éditeur}

Presses universitaires de Rennes

Édition imprimée

Date de publication : 6 décembre 2018

Pagination : 129-154

ISBN : 978-2-7535-7572-1

ISSN : 0399-0826

\section{Référence électronique}

Jean-Philippe Priotti, «Au cœur des échanges européens : argent américain et circuits économiques entre la Bretagne et l'Espagne (1570-1635) », Annales de Bretagne et des Pays de l'Ouest [En ligne], 125-3 | 2018, mis en ligne le 06 décembre 2020, consulté le 06 janvier 2021. URL : http:// journals.openedition.org/abpo/3958; DOI : https://doi.org/10.4000/abpo.3958 


\title{
Au cœur des échanges européens : argent américain et circuits économiques entre la Bretagne et l'Espagne (1570-1635)
}

\author{
Jean-Philippe PRIOTTI \\ Maître de conférences en histoire moderne, Université Lille Nord de France - \\ ULCO, HLLI (Boulogne-sur-Mer)
}

Dans les lignes qui suivent, il s'agit d'examiner les conséquences des arrivées massives de métaux précieux (1570-1640) sur différents circuits commerciaux et marchés qui lièrent la Bretagne à la Castille et à l'Andalousie' ${ }^{1}$.

Si à cette époque les entrepreneurs malouins, vitréens et morlaisiens comptent parmi les plus actifs de Bretagne, c'est qu'ils possédaient déjà une solide expérience dans différents domaines. Des pêcheries de TerreNeuve et des entreprises de découverte au Canada à la course, en passant par leur activité d'armateurs, de transporteurs et de marchands de toiles et de draps dans les îles proches et en Angleterre, ils avaient multiplié des expériences de diverse nature et interactives autour de la pêche, du commerce et de la guerre, et avaient réussi à accumuler des capitaux ${ }^{2}$. À la

1. Cet article résulte de l'approfondissement et de l'élargissement chronologique de deux publications : "Metales preciosos, competencia comercial y transformación económica en el Atlántico franco-español (1550-1570) ", Jahrbuch für Geschichte Lateinamerikas, 43, Böhlau-Verlag-Köln-Weimar-Wien, 2006, p. 25-40; "Logiques commerciales d'une globalisation. Les toiles françaises dans l'Atlantique hispano-américain (1550-1600) ", dans PÉREZ, Béatrice, Rose, Sonia et ClÉMENT, Jean-Pierre (dir.), Des marchands entre deux mondes, Paris, Presses Universitaires de la Sorbonne, 2007, p. 15-41.

2. En 1541, on armait à Saint-Malo, à la demande du roi de France, 13 navires avec une bonne artillerie et tous les équipements, munitions et maintenance pour plus de deux ans. Jacques Cartier, d'origine malouine, commandant l'opération, allait peupler les nouvelles terres avec 2500 hommes. Parmi les navires, certains étaient armés pour aller aux pêcheries de Terre-Neuve, Archivo General de Indias-Patronato, 267, no 1, r13/1/1. Entre 1546 et 1550 , des corsaires de Saint-Malo capturèrent deux navires d'une valeur de 4000 ducats, AllOZA APARICIO, Ángel, Europa en el mercado español. Mercaderes, represalias y contrabando en el siglo XVII, Salamanca, Junta de Castilla y León, 2006, appendice 1 p. 235. Dans l'état des dépenses de la flotte du Ponant fait en 1636, sur l'ordre de Richelieu, apparaît "La perle ", frégate malouine de 300 tonneaux commandée par le sieur de Boisjoly, 
faveur du soulèvement des Pays-Bas du Nord et des guerres entre l'Espagne et l'Angleterre, les Bretons accentuèrent leur participation au transport de marchandises anglaises et hollandaises vers la Péninsule, tant que durèrent ces guerres et avant que n'éclatent les conflits entre la France et l'Espagne en 1635 .

Leur présence dans la Monarchie hispanique se renforça dans les années 1560-1570 et cette condition de transporteurs fut conduite parallèlement à des activités plus strictement commerciales. Dans les années 1570, mis à part le blé et les toiles vendus en Andalousie, ils agissaient en Biscaye, au Portugal, aux Canaries et en Méditerranée ${ }^{3}$. À Carthagène, ils étaient présents dès les années 1570 où ils agissaient comme marchands de toiles et de produits du nord de l'Europe et de la Baltique ${ }^{4}$. À la fin du Xvi ${ }^{\mathrm{e}}$ siècle, ils amarraient leurs frégates à Civitavecchia et même en Méditerranée orientale. Également tournés vers des horizons lointains, ils organisaient des voyages directs vers l'Amérique espagnole au début du XvII ${ }^{\mathrm{e}}$ siècle et fondaient des compagnies pour aller faire le commerce des peaux au Canada ${ }^{5}$.

de Saint-Malo, Correspondance de Henri d'Escoubleau de Sourdis : augmentée des ordres, instructions, et lettres de Louis XIII et du Cardinal de Richelieu à M. de Sourdis concernant les opérations des flottes françaises de 1636 a 1642, Paris, Imprimerie de Crapelet, 1839, vol. 1, p. 40.

3. À partir des années 1570, on voyait des navires malouins ancrés à Bilbao : marchands malouins et de Pontivy y vendaient leurs toiles, voir annexe 3 de PrIOTTI, JeanPhilippe, Bilbao et ses marchands : genèse d'une croissance, Villeneuve-d'Ascq, Presses du Septentrion, 2004 et aussi Archivo Foral de Bizkaia, judicial, 1277/094, 4 décembre 1567, Archivo Foral de Bizkaia, corregimiento, 1039/053; Archivo Histórico Provincial de Bizkaia, leg. 3866, 3869, 3871. Au début du XVII ${ }^{\mathrm{e}}$ siècle, on les trouvait aussi à Nantes affretant et assurant des navires pour l'Espagne, TANGUY, Jean, Le commerce nantais à la fin du XVI eiècle et au début du XVII siècle, Rennes, thèse de doctorat, 1967, t. II, p. 296; Archivo Histórico Provincial de Bizkaia, Leg. 3857, leg. 3858, leg. 4052. De même, à cette époque, ils continuent à fréquenter les ports du Nord de l'Espagne : à Bilbao, le 12 janvier 1609, Alonso Brinon arrive de Saint-Malo sur son navire; le 6 février 1609, c'est le tour du Claude, maître Jacques Brebas, de Saint-Malo; le 23 mars 1609 arrive à Bilbao le navire Pedro de Saint-Malo; le 27 mars 1609, la María Rosa, de Saint-Malo, maître Alonso Brinon; le 27 mars la Française, de Saint-Malo, maître Jacques Thomas; le 19 octobre 1609, la María Rosa, de Saint-Malo, maître Alonso Tuñon; le 26 mars 1610, la Francesa de Saint-Malo, maître François Thomas; le 23 août 1610, la Francesa, de Saint-Malo, François Thomas; le 11 septembre 1610, la flor de Lis, de Saint-Malo, maître Olivier Rui; le 3 décembre 1610, Le Jacques, maître Philippe Jales qui arrive de Saint-Malo; le 3 février 1611, la Fleur de Lys, de Saint-Malo, maître Olivier Genu, le 16 février 1611, le Charles, maître Jean Bernal, de Saint-Malo; le 3 mars 1611, le Jacques de Saint-Malo, maître Philippe Jas qui est venu de Saint-Malo; le 21 avril 1611, le Jacques, Philippe Jos, arrive avec des ballots; le 15 juillet 1611, le Crésus, Jacques Rabino que vient de Saint-Malo; le 20 juillet 1611, le Jacques, maître Philippe Jas que vient de Saint-Malo, Archivo Foral de Bizkaia, libro de averías, 1609-1612.

4. Montojo Montojo, Vicente et Ruzz IbáÑEz José Javier, « Las comunidades mercantiles genovesa y malouina en Cartagena (siglos XVI-XVII) ", dans MoTTA, Giovanna (dir.), Mercanti e viaggiatori per le vie del mondo, Milano, 2000, p. 75-90.

5. Les Malouins se lanceraient dans des relations directes avec les Indes occidentales et orientales au début du XVII ${ }^{\mathrm{e}}$ siècle, TANGUY, Jean, Le commerce nantais à la fin $d u$ XVI siècle..., op. cit., 1967, t. II, p. 301. Une compagnie de de 90000 livres est créée en 1604 pour le commerce avec le Canada dans laquelle participaient des Malouins pour les 2/5 
Nous allons apprécier jusqu'à quel point la géographie, la variété et le volume des affaires traitées permettent de les considérer comme d'authentiques entrepreneurs. Car, au-delà de la bibliographie existante pour une période postérieure, le développement des affaires des Bretons du Nord, leur caractère entreprenant et leur longue trajectoire économique en Espagne, ressortent de plusieurs sources ${ }^{6}$.

À partir des années 1560-1570, au moment de l'augmentation des arrivées de métaux précieux en Andalousie et de la paix entre la France et l'Espagne (1559-1635) - en dehors de la période 1595-1598 - eurent lieu des changements significatifs dans les relations commerciales atlantiques entre les deux royaumes. En Espagne, la plus grande disponibilité de métal provoqua la polarisation du commerce castillan par les ports andalous et promut aussi le développement d'échanges avec certains ports français (dont SaintMalo) à un niveau remarquable. L'afflux d'argent aiguisa la concurrence entre différents groupes marchands et leur organisation commerciale.

Grâce à l'introduction de l'amalgame dans le processus de production de l'argent américain et aux quantités croissantes de métal parvenues en Andalousie, les années 1560-1570 marquent le passage d'une économie dont l'axe principal connectait le nord de la Castille au reste de l'Europe à travers les ports vasco-cantabriques, à une économie contrôlé par Séville et ses avant-ports, grâce à son commerce européen et américain ${ }^{7}$. Ce changement engendra un certain renouvellement des forces commerciales. En

(l'un d'eux se nommait Du Pont-Gravé), des Rouennais pour $1 / 5^{\mathrm{e}}$, des Rochelais pour $1 / 5^{\mathrm{e}}$, le dernier cinquième échoyant à De Monts, Trocmé, Émile et Delafosse, Marcel, $L e$ commerce rochelais de la fin du XV siècle au début du XVII e, Paris, A. Colin, 1952, p. 166-167. Ils participaient également avec leurs associés de Vitré, Dinan et Laval à une expédition aux îles Moluques qui se solda par un échec. Frotet et Grout, deux habiles malouins, commandaient "Le Corbin " et " le Croissant ", Frain DE LA GaulaYrie Édouard, Les Vitréens..., op. cit., p. 51. Voir aussi dans ce volume la contribution de Guillaume Lelièvre.

6. À défaut de matériaux archivistiques consistants (de type privé ou officiel, en France et en Espagne) pour Saint-Malo au Xvi ${ }^{\mathrm{e}}$ siècle, il s'avère difficile d'apprécier le volume des négoces que les Malouins brassaient. Nous avons donc eu recours aux correspondances diplomatiques de l'ambassadeur français à Madrid, à la documentation de l'Archivo General de Indias ainsi qu'aux protocoles notariés de Cadix mis à part d'autres sources secondaires.

7. On s'occupera plus en détail - mais pas exclusivement - de la commercialisation des toiles françaises (en particulier bretonnes), marchandise la plus vendue en Castille pendant les années 1560-1570 et qui représentait aussi à cette époque le gros des chargements destinés au Nouveau Monde, LoRENzo SANz Eufemio, Comercio de España con América en la época de Felipe II, 2 vol., Valladolid, Diputación Provincial de Valladolid, 1979-1980 et PRIOTTI, Jean-Philippe, Bilbao y sus mercaderes en el siglo XVI. Génesis de un crecimiento, Bilbao, Diputación Foral de Bizkaia, 2005. Au xvII ${ }^{\mathrm{e}}$ siècle, ce produit continua à tenir une place importante, GIRARD, Albert, El comercio francés en Sevilla y Cádiz en tiempos de los Habsburgo, Sevilla, Renacimiento, 2006 ( $1^{\text {re }}$ éd. en français 1932); MoRINEAU, Michel, Incroyables gazettes et fabuleux métaux, Londres/Paris, Cambridge University Press/Éd. de la Maison des Sciences de l'Homme, 1985; Bustos Rodríguez, Manuel, Cádiz en el sistema atlántico. La ciudad, sus comerciantes y la actividad mercantil (1650-1830), Cádiz, Sílex, 2005. 
effet, les Castillans - comme la famille Ruiz que nous étudierons en détail ${ }^{8}$ qui contrôlaient une bonne part des échanges du nord de la Castille avec Nantes, échouèrent à étendre leur influence vers Séville et l'Amérique. Ils furent jusqu'à un certain point évincés par des marchands originaires du nord de la Bretagne (Saint-Malo, Vitré, Morlaix, Pontivy) qui réussirent à s'imposer dans les échanges entre la Bretagne et l'Espagne, en plaçant leurs intérêts en Andalousie. Cette captation commerciale effectuée par les Bretons du Nord coïncida avec l'afflux de métaux précieux et avec la paix du Cateau-Cambrésis signée avec la France, principal partenaire commercial du voisin espagnol ${ }^{9}$.

La dynamique compétitive est sans doute une des clés qui permet de mieux comprendre l'expansion de la puissance européenne en Atlantique et ce processus constituerait même - selon certains auteurs - un des principaux avantages de l'Occident sur le reste du monde ${ }^{10}$. Il est donc particulèrement intéressant d'essayer de détecter la façon dont le rapport de force se modifia entre les trois groupes auxquels nous venons de faire référence : Castillans, Andalous et Bretons.

\section{Processus de conquête malouin des marchés espagnols}

À la fin des années 1550, les frères Ruiz (André et Simón) et leurs associés, établis respectivement à Nantes et à Medina del Campo, contrôlaient une bonne part des échanges entre la Bretagne et la Castille ${ }^{11}$ et, partant, ils s'impliquèrent dans la distribution de produits jusqu'en Andalousie. Malheureusement pour eux, les bénéfices juteux produits par les ventes en Castille n'eurent pas d'équivalent à Séville et les négoces andalous ne produisirent jamais de bons résultats, en particulier à cause des mauvaises "ditas ${ }^{12}$ ".

Durant les années 1560 et plus encore par la suite, de nouveaux commerçants venus du nord de la Bretagne, principalement de Saint-Malo et de Vitré - qui jusqu'alors n'avaient pas accosté aussi fréquemment sur

8. Cette firme avait des affaires en Bretagne, en Castille et en Andalousie. Voir le travail de LAPEYRE, Henri, Une famille de marchands : les Ruiz, Paris, A. Colin, 1955.

9. En 1624, un mémoire français signale les toiles comme étant l'article le plus demandé en Amérique et indiquait qu'il ne se fabriquait plus qu'en France, puisque la Hollande, la Flandre et autres provenances n'en envoyaient pas beaucoup au Nouveau Monde, GIRARD, Albert, El comercio..., op. cit., p. 314.

10. Ferguson, Niall, Civilization. The West and the Rest, éditions Allen Lane, 2011.

11. Voir les droits de douanes des dîmes de la mer payés en 1559-1560 par Simón Ruiz, ses employés et associés publiés par LAPEYRE, Henri, El comercio exterior de Castilla a través de las aduanas de Felipe II, Valladolid, Universidad de Valladolid, 1981, p. 79-80.

12. La correspondence de Francisco de Mariaca, Llorente de Angulo et Jerónimo de Valladolid (installés à Séville) à Simón Ruiz, de Medina del Campo, donne un bon exemple des problèmes auxquels était confronté le Castillan pour encaisser l'argent des marchandises vendues à crédit aux cargadores sévillans dans les années 1560-1570. De ces inconvénients émanèrent de nombreux procès, parfois interminables, et presque toujours très coûteux, Archivo Histórico Provincial de Valladolid : fonds Ruiz. 
le littoral ibérique - multiplièrent les liaisons maritimes entre la Bretagne et l'Andalousie avec de nombreuses marchandises à bord ${ }^{13}$. Après les avoir vendues au comptant à Sanlúcar de Barrameda et à Cádiz, ces marchands itinérants retournaient en France avec des barres et/ou des pièces d'argent ${ }^{14}$. Contrairement à ce qui se passait pour le commerce entre le nord de la Castille et le reste de l'Europe, dans ces échanges dominaient les paiements au comptant ${ }^{15}$. L'augmentation de la production d'argent péruvien et de son exportation vers l'Andalousie dans les années 1570, son maintien à un haut niveau - malgré des variations - jusqu'aux années 1630, fit croître et soutint la vigueur des échanges commerciaux pour lesquels on recourrait assez peu aux foires de change. En témoignent d'une certaine façon les grandes quantités d'argent qui prirent le chemin des ports bretons, de celui de Saint-Malo en particulier ${ }^{16}$.

Dès le début de la période considérée, l'usage du paiement métallique provoqua trois types de conséquence pour les firmes castillanes qui travaillaient majoritairement à crédit, soldant leurs achats aux foires de Lyon.

13. À cette époque, la solidarité entre Vitréens et Malouins était évidente. Après le séquestre de plusieurs navires avec de l'argent à leur bord, les Malouins prirent la mer avec 12 navires et récupérèrent des Rochelais la majeure partie des prises qui avaient été faites à leurs dépens, Archivo Histórico Provincial de Valladolid, fonds Ruiz, C27-230, Julien Ruiz à Simón Ruiz, 24 avril 1575.

14. S'agissant de contrebande - au moins en ce qui concerne les sorties d'argent de la Péninsule - ce commerce d'argent a laissé peu de traces dans les archives. Comme le suggère Antonio Miguel Bernal l'échange d'argent-métal contre des marchandises n'était pas instantané et le paiement devait s'effectuer à travers des cédules ou des vales de banco, c'est-à-dire de lettres qui signifiaient un crédit à court terme.

15. L'opposition entre crédit et argent comptant est délicate et exige quelques précisions. Bien entendu, aucun circuit marchand ne fonctionne sans l'apparition de comptant à un moment ou à un autre. Et le paiement au comptant est rarement instantané ne seraitce que parce que l'acheminement de la marchandise et son conditionnement requièrent un délai, même court avant que l'échange (surtout s'il représente de grandes quantités) soit effectivement réalisé. Cela étant, l'argent n'était pas seulement un instrument de paiement mais aussi une marchandise dotée d'une valeur qui variait d'un lieu à l'autre. En ce sens il était plus aisé d'en faire commerce que de spéculer sur le change. Il est probable que le commerce au comptant opéré par les Malouins resta majoritaire par rapport à un commerce à crédit (par lettres de change) dont on a peu de traces jusqu'au XvII ${ }^{\mathrm{e}}$ siècle. Mais même si les Malouins et leurs associés parvinrent à participer à des opérations de crédit, ils n'avaient pas l'habitude de fréquenter les foires de change. A. Girard fait mention d'une opération de change qui eut lieu vers 1660. En raison de la difficulté à placer leurs marchandises à cause de la concurrence, les marchands français innovèrent avec un nouveau procédé. Au lieu de vendre au comptant, ils vendaient en partie au comptant et en partie à $12 \%$ d'intérêt payable au retour de la flotte, op. cit., p. 407. Wilfrid Brulez confirme l'importance de l'usage du comptant au xvI ${ }^{\mathrm{e}}$ siècle. Selon lui, c'est même un des traits caractéristiques de l'époque perturbée de la seconde moitié du Xvi siècle, à la place de la vente à crédit, De firma della Faille en de internationale handel van vlaamse firma's in de $16^{e}$ eeuw, Brussel, Paleis der Academièn, 1959, p. 581.

16. Pour le volume d'argent produit en Amérique et les arrivées à Séville, on peut consulter, Morineau, Michel, Incroyables gazettes ..., op. cit., p. 78-82; STEIN, S.J., STEIN, Barbara H., Silver, Trade and War. Spain and America in the Making of Early Modern Europe, BaltimoreLondon, The Johns Hopkins University Press, 2000, p. 24. Pour les quantités arrivées à Saint-Malo, voir l'annexe 1. 
Sous la pression d'un nombre plus important d'acheteurs sur les marchés bretons, le prix des toiles augmenta ${ }^{17}$. Ces concurrents du nord de la Bretagne ne se limitèrent pas à faire augmenter les prix : usant d'argent comptant, ils réussirent à se réserver les produits les plus chers et de meilleure qualité, comme les "bretagnes ", les " lavals " et en profitèrent aussi pour accéder aux marchés normands voisins ("rouens"). Pour autant, ils ne délaissèrent pas les toiles de moindre valeur (" angers ", "brins " et "mélinges "). De ce fait, ils obligeaient les facteurs des Ruiz et d'autres Castillans à revenir bredouille des foires bretonnes, à opter pour des produits de qualité et/ou plus à se replier sur les marchés au sud du duché, près de Nantes. Manifestement, les acheteurs munis d'argent comptant se taillaient la part du lion dans le marché des toiles bretonnes ${ }^{18}$.

Il en resulta une relative spécialisation des marchés espagnols (l'andalou et le castillan) en fonction de deux usages distincts de la monnaie : en faveur du marché andalou et des Bretons du Nord, et aux dépens du marché castillan et des Castillans établis dans le sud de la Bretagne, relativement évincés. Phénomène durable puisqu'à la fin du XvII ${ }^{\mathrm{e}}$ siècle, les toiles françaises les plus fines continuaient à se vendre en Amérique et les plus grossières en Espagne, les Malouins contrôlant toujours la production bretonne au grand dam des Nantais ${ }^{19}$.

En conséquence, des changements avaient aussi lieu en France, car les envois que les Bretons du Nord effectuaient en Andalousie étaient surtout réalisés via le port de Saint-Malo et non plus via celui de Nantes, ce qui tendait à déplacer la zone de plus grande activité du sud de la Bretagne vers le Nord ${ }^{20}$. L'utilisation d'argent-métal permit aux Malouins de contrôler la production, contrôle facilité par ailleurs par la proximité des centres de production, élément essentiel en période d'abondance monétaire et de concurrence accrue.

Ce processus concurentiel montre que l'initiative commerciale avait changé de mains. En accentuant leur contrôle de la production, les Bretons

17. De nombreuses allusions à cette hausse des prix se trouvent dans la correspondance d'André Ruiz, père, de Julien Ruiz et d'Yvon Rocaz pour les années 1560-1570, Archivo Histórico Provincial de Valladolid : fonds Ruiz.

18. Sur cette question, GRENIER, Jean-Yves, L'économie d'Ancien Régime. Un monde de l'échange et de l'incertitude, Paris, Albin Michel, 1996.

19. GiRARD, Albert, op. cit., p. 322. Les négociants de Nantes se trouvaient trop loin des zones de production des " bretagnes " et ne pouvaient pas surveiller la qualité de la marchandise, de sorte que souvent ils se retrouvaient avec des toiles de piètre qualité. Ils devaient laisser aux gens de Morlaix et Saint-Malo les meilleurs textiles, ZYLBERBERG, Michel, Une si douce domination. Les milieux d'affaires français et l'Espagne vers 1780-1808, Paris, Ministère des Finances, 1993, p. 201.

20. De fait, au XVII ${ }^{\mathrm{e}}$ siècle, on peut observer deux phénomènes : le renforcement de la présence de navires malouins à Bilbao, aux dépens de ceux des autres flottes bretonnes, en particulier de celle de Nantes et des ports environnants, GUIARD-LARRAURI, Teófilo, Historia del Consulado de Bilbao y Casa de Contratación de Bilbao y del comercio de la villa (1511-1699), Bilbao, José de Astuy, 1913, p. 400 et suivantes, et aussi l'augmentation de la présence vitréenne et malouine à Nantes. 
du Nord allaient s'imposer sur les circuits commerciaux. L'arrivée des Vitréens et des Malouins en Andalousie (surtout à Cadix et à Sanlúcar) qui vendaient au comptant soulevait plusieurs problèmes. Comme ils souhaitaient se défaire rapidement des toiles, ils mettaient en vente à bas prix portant ainsi préjudice aux Castillans qui eux aussi vendaient à Séville ${ }^{21}$. Offrant de meilleures conditions de vente et vendant de la marchandise de qualité, ils accaparaient l'argent-métal ou le comptant laissant aux Castillans l'épineux problème du crédit, sur un marché à hauts risques comme l'était le marché sévillan.

En conséquence, les Castillans, comme les Ruiz, perdirent de l'argent sur ce marché où les marges bénéficiaires étaient potentiellement plus hautes qu'en Castille, compte tenu de la plus grande élasticité des prix en Andalousie, dont les connexions américaines offraient des gros gains, insoutenables sur le marché castillan. En effet, la hausse des prix survenue en France due à une plus forte demande sur les marchés d'achat, pouvait être répercutée plus facilement sur les prix de vente en Amérique que sur les prix en vigueur en Castille. André Ruiz (le frère de Simón) établi à Nantes, comprenait bien le processus de concurrence en cours au début des années 1560. Il écrit à son frère : "En Laval todo se ha vendido : han comprado [...] para los que van a Andalucía. Y también en Rouen ha sido lo mismo. La ganancia no es sino para los que negocian otramente ${ }^{22}$. " En 1563, il conseillait même à son frère d'agir à Séville comme ceux de Vitré et de Saint-Malo, voyageant avec la marchandise et payant au comptant ${ }^{23}$. D'ailleurs, à Nantes comme à Orléans, à Lyon et à Paris, des marchands faisaient de même ${ }^{24}$.

Cette lutte entre Castillans et Bretons du Nord pour l'obtention de toiles de Bretagne avait des répercussions importantes dans l'organisation des firmes castillanes, et modifiait non seulement la teneur des liens des Ruiz entre eux mais aussi ceux de la communauté d'intérêts à la tête de laquelle se trouvait la firme au début des années 1560. Ces tensions

21. Vendre la marchandise bon marché en Andalousie n'était pas un inconvénient pour eux, puisque s'ils perdaient de l'argent en vendant la marchandise, ils compensaient ces pertes éventuelles par la vente des réaux et des barres d'argent très bien cotés en France. $\mathrm{Au} \mathrm{XVI}{ }^{\mathrm{e}}$ siècle, les frappes monétaires à Rennes, approvisionnées à partir de Saint-Malo, attinrent leur niveau maximum entre 1571 et 1590, période pendant laquelle elles dépassèrent celles de Nantes, SPOONER, Frank C., L'économie mondiale et les frappes monétaires en France, 1493-1680, Paris, Armand Colin, 1956, cartes, p. 263-264.

22. Archivo Histórico Provincial de Valladolid, C14-256, André Ruiz à Simón Ruiz, 5 décembre 1571. Un an et demi plus tard, il réitérait : "Este trato no es para vuestra merced, ni para nosotros, sino para los que tratan de otra manera que hacemos nosotros, que tienen cada día dinero".

23. Archivo Histórico Provincial de Valladolid, C2-117 André Ruiz à Simón Ruiz 7 avril 1563. La correspondence d'Yvon Rocaz et de Julien Ruiz donnent à observer les mêmes mécanismes.

24. Il descend à Saint-Malo, affirmait le procureur des bourgeois de Saint-Malo «nombre d'argent d'Espagne appartenant à d'autres marchands de Lyon, Paris, Orléans. Ses habitants le transportent en leurs maisons et s'en instituent les courtiers..." 
débouchèrent sur une perte de contrôle comercial de la firme Ruiz sur l'axe Castille-Nantes au cours des années 1565-1575 et coïncidèrent aussi avec un intérêt plus marqué de Simón Ruiz pour les activités financières, tandis que certains Nantais - imitant la façon de faire des Malouins et des Vitréens - optaient pour le commerce maritime avec l'Andalousie ${ }^{25}$. La rivalité entre Malouins et Castillans avait donc provoqué, en partie au moins, un autre processus concurrentiel entre les anciens partenaires nantais et castillans. Il s'agissait en fait d'un changement de grande ampleur : à une phase durant laquelle les Castillans avaient essaimé dans toute l'Europe dès la fin du Xve siècle succédait une phase de repli vers la Péninsule et, en même temps, une conquête commerciale en sens inverse promue par les Français. L'abondance d'argent américain jouait un rôle de premier plan dans ce changement structurel.

Jusqu'aux années 1560-1570, afin de monopoliser l'activité d'intermédiation entre l'offre sur les marchés bretons et la demande castillane, les Ruiz avaient contrôlé horizontalement le marché créant des firmes connexes grâce à leurs capitaux et au crédit. Mais dès l'arrivée de concurrents ils avaient pâti des effets d'une nouvelle forme d'organisation basée sur une intégration verticale des activités et s'appuyant sur l'argent-métal qui leur permettait de contrôler la production, le transport - nombre de ces marchands ambulants (dans un premier temps) étaient propriétaires et/ou capitaines des navires à bord desquels ils voyageaient, ou appartenaient à des familles d'armateurs - et la vente, sans que la marchandise change de mains, c'est-à-dire sans nécessité de relais commerciaux, opérant un commerce de port en port. Cette logique excluait les concurrents d'autres nations et était le moyen priilégié pour s'imposer sur de nouveaux marchés à coût réduit.

Dans ce cadre compétitif, pour pouvoir s'imposer, il était nécessaire de réduire une série de coûts et de risques. En se limitant à un commerce portuaire, la circulation de la marchandise et la rotation du capital s'accéléraient, ce qui permettait de faire des affaires plus librement, pendant que les villes de l'intérieur, surtout celles où se tenaient les foires, étaient soumises à de multiples contraintes de diverse nature, de la part du pouvoir royal notamment ${ }^{26}$. De même, grâce à leur capacité d'adaptation rapide

25. Pour plus de détails sur ce processus, je me permets de remmettre le lecteur à mon travail : "Metales preciosos... ", p. 25-40. À la fin du XVI ${ }^{\mathrm{e}}$ siècle et dans la première moitié du siècle suivant, des Nantais firent leur apparition parmi les Français établis en Andalousie, comme les membres de la famille Poullain qui firent partie du consulat français de Séville dans les années 1620, LoRENzo SANZ, Eufemio, Comercio..., op. cit., t.I p. $91 s q q$.

26. Le cas de Medina del Campo, par exemple, a été traité par ABED AL-Hussein, Falah H., Trade and business community in Old Castile. Medina del Campo, 1500-1575, thèse de l'université d'East Anglia, 1982. Dans les années 1570, aussi bien en Espagne qu'en France, la négociation des marchandises eut chaque fois davantage lieu dans les ports de l'Ouest, voir EsPEJo, Cristóbal et PAZ, Julián, Las antiguas ferias de Medina del Campo, Valladolid, Editorial Maxtor, 2003 (1 ${ }^{\text {re }}$ éd. 1908), p. 69, 72, 311. Dans une lettre à Simón Ruiz, Antonio Gomes écrivait que l'absence de droits à payer en Biscaye invitait les gens à aller y faire 
aux changements politiques ou parce qu'ils étaient situés en dehors de la juridiction royale, les ports avec accès direct à la mer pouvaient déjouer les embargos sur l'argent des particuliers et certaines taxes d'État. C'était l'avantage de Cadix et de Sanlúcar par rapport à Séville, et aussi celui de Saint-Malo, port-franc, par rapport à Nantes et même à Rouen. La volatilité de l'économie commerciale résultait souvent de cette tension entre les logiques des acteurs commerciaux et celles des gouvernements.

Le commerce effectué en dehors de Séville et des circuits légaux devait beaucoup à ce qu'une bonne quantité d'argent arrivait d'Amérique sans avoir payé le quinto real ${ }^{27}$. Comme cet argent ne pouvait pas être déclaré sans courir un certain risque, il était plus facile et moins coûteux d'établir son négoce à Sanlúcar et Puerto Santa María, où respectivement les ducs de Medina Sidonia et de Medinaceli protégeaient les étrangers. On payait également moins de droits à Cadix qu'à Séville ${ }^{28}$. L'économie de coûts entre le système au comptant et le système utilisant le crédit se manifestait aussi - de façon assez paradoxale - par un prix de transport modeste de l'argent. Malgré le danger couru, transporter de l'argent-métal de Cadix à Saint-Malo coûtait entre 1,5 et $2 \%{ }^{29}$. Par conséquent, il était moins coûteux pour un vendeur de traiter avec un acheteur en possession d'argent sonnant que de vendre à tempérament avec paiement à l'échéance d'une foire, surtout si l'on tient compte des problèmes de solvabilité de nombreux cargadores dans les années 1564-1569 et 1573-1576 ${ }^{30}$. Le commerce itinérant que menaient Vitréens et Malouins, de port à port, permettait également d'économiser une commission de 1,5 ou $2 \%$ que l'on payait d'ordinaire aux intermédiaires établis aux points de rupture. Le fait de multiplier les points de charge et décharge, et d'allonger les circuits commerciaux augmentait

leurs achats et leurs ventes, lettre 87, Antonio Gomes à Simón Ruiz, 2 février 1575, publiée par GENTIL DA SiLVA, José, op. cit., t.II, p. 89-90.

27. Archivo General de Indias-Contaduría 1775, numéro 13. En 1584, dans un mémoire de la "plata y oro que hay en el Perú ", on mentionnait les quantités croissantes d'argent qui arrivaient en Andalousie bien que le quint royal restât stable. Girard affirmait que les marchands ne déclaraient jamais plus de la moitié de ce qu'ils apportaient en argent et en marchandises, GIRARD Albert, El comercio..., op. cit., p. 184-185.

28. Des calculs rapides faits pour le XVII ${ }^{\mathrm{e}}$ siècle donnent une idée claire de l'avantage relatif de Cadix et d'autres ports de la côte andalouse vis-à-vis de Séville. En 1635, le total s'élevait à $19 \%$ pour les douanes sévillanes au lieu de 7,5\% pour Cadix, GIRARD, Albert, El comercio..., op. cit., p. 199. Et les possibilités de contrebande dans la baie ou encore à Puerto Santa María et Sanlúcar réduisaient davantage encore les coûts de transaction.

29. GIRARD Albert, op. cit., p. 408. Le fret de l'argent au départ de Cadix était meilleur marché sur les navires marchands hollandais, à hauteur de $1 \%$ seulement, GIRARD, Albert, op. cit., p. 428. Lorsqu'on embarquait l'argent-métal à bord d'un navire de guerre, il en coûtait $2 \%$ car le voyage vers les ports européens était alors très sûr. Dans le commerce d'Espagne, on assurait peu le métal, car les frégates étaient toujours armées comme en période de guerre.

30. Je renvoie ici aux lettres envoyées à Simón Ruiz depuis Séville dans ces années-là, dans lesquelles sont référencées les nombreuses faillites ayant lieu dans la ville; voir aussi LORENZO SANZ, Eufemio, op. cit., t.1, p. 182-207. 
aussi les risques (intempéries, vols, pertes, etc.) sans qu'un meilleur profit soit pour autant assuré.

L'économie relative de coût se combinait avec des bénéfices potentiellement élevés sur l'itinéraire entre Saint-Malo et l'Andalousie vue la rapidité des opérations commerciales et le prix de l'argent-métal en France. Un voyage aller-retour Saint-Malo-Cadix-Saint-Malo se faisait en quelques mois. Compte tenu de la croissance de la demande andalouse et américaine, Malouins et Vitréens faisaient parfois deux voyages par an, ce qui expliquait par ailleurs l'augmentation du prix des toiles et du blé en France ${ }^{31}$. C'était un commerce profitable : des ventes de blé à Sanlúcar en 1605 - effectuées en l'espace de quatre mois - donnaient des profits nets de $60 \%{ }^{32}$. Ces bénéfices n'avaient rien à envier à ceux qu'Espagnols et Mexicains obtenaient dans le commerce transatlantique à plus longue distance ${ }^{33}$. Dès la fin du XvI ${ }^{\mathrm{e}}$ siècle, le succès des Malouins et des Vitréens était notoire : ils contrôlaient bonne part du commerce avec Sanlúcar, Cadix et les Canaries et l'on trouvait parmi eux des hommes d'affaires trois fois plus riches que ceux de Nantes ${ }^{34}$.

Mis à part ce qui pouvait être gagné en vendant des toiles ou du blé en Andalousie, la vente des barres d'argent-métal en France rapportait des plus-values qui oscillaient entre 32 et $250 \%$ dans les années $1570^{35}$. Des

31. Archivo Histórico Provincial de Valladolid-C51-31, Francisco Morovelly à Simón Ruiz, 4 janvier 1579 .

32. Il s'agissait de 440 fanègues, FrAIn DE LA GAULAYRIE, Édouard, Les Vitréens..., op. cit., p. 657.

33. Voir Bernal, Antonio Miguel, España, proyecto inacabado. Los costes/beneficios del Imperio, Madrid, Marcial Pons, 2005, p. 493 et suiv.; Schell Hoberman, Louisa, Mexico's Merchant Elite, 1590-1660, Durham-London, Duke University Press, 1991, p. 54, 60. Les gains des chargeurs sévillans - on ne sait si les dépenses sont comptées ou pas - étaient pour les chargements de Terre Ferme de 1564 de 120 \% et pour le Pérou en 1568, entre 70 et $90 \%$. Quant aux marchands de México, ils obtenaient des bénéfices oscillant entre 7 et $50 \%$ dans le commerce transatlantique de la première moitié du XVII ${ }^{\mathrm{e}}$ siècle.

34. Archivo Histórico Provincial de Valladolid-C3-229, Yvon Rocaz à Simón Ruiz, 10 janvier 1565. "Algun día, hemos de probar el camino donde van los de Vitré, por los ver venir con mucho dinero y ser ricos ", cité par LAPEYRE, Henri, Une famille..., op. cit., p. 121. Dans le dernier tiers du XVI ${ }^{e}$ siècle, la richesse des grands commerçants malouins permit les premières tranformations urbaines de l'enceinte médiévale de la ville et la construction de nombreuses maisons et d'hôtels particuliers en pierre fut accomplie dans les années 1590-1620, Petout, Philippe, Hôtels et maisons de Saint-Malo, XVI ${ }^{e}$-XVII - -XVIII ${ }^{e}$ siècles, Paris, Picard, 1985, p. 17, 42-43.

35. Au debut des années 1570, malgré les pertes de 12 à $15 \%$ sur la vente des marchandises françaises en Andalousie, les Bretons faisaient des bénéfices grâce à la vente de l'argent-métal et des réaux en France. Entre 1570 et 1577, la valeur du réal oscillait entre 4 sous 6 deniers et 12 sous, c'est-à-dire entre 45 et 120 maravédis tandis qu'en Castille le réal valait 34 maravedís, ce qui équivalait à un gain de $32 \%$ à $250 \%$. Les marchands s'exprimaient sans détour (C12-159 André Ruiz à Simón Ruiz mars 1570) : "La ganancia no es sino para los que negocian otramente " [...] "Este tiempo no es como los pasados para haber telas. Y hay tantos compradores. Este negocio no es sino para los que traen el real que vale 4 sueldos 6 dineros que ya entran con 31 [\%] de ganancia en el cambio y asî a cualquier precio que vendan la mercancía no pueden dejar de ganar mucho ". C 20-122 31-01-1573 André Ruiz à Simón Ruiz : "El escudo pasa a Nantes a $54 \$$ y el real de a 4 a $4 \$ 7$ 
Bretons faisaient de même au Portugal ${ }^{36}$. Bien que ce pourcentage ait sans doute varié dans l'intervalle 1570-1630, le profit sur la vente d'argent américain fut toujours de mise, en France ou dans les autres contrées du nordouest européen ${ }^{37}$. À la fin du XVI ${ }^{\mathrm{e}}$ siècle, divers témoignages évoquaient la fuite des barres d'argent de Saint-Malo vers l'Angleterre et la Hollande, territoires où l'on payait davantage pour lui qu'en France. Lorsque c'était l'or qui rapportait de gros profits, les plateros français installés en Andalousie changeaient l'argent pour l'or, et l'envoyaient ensuite en France. Le fret de retour n'était pas seulement constitué de métaux précieux. À SaintMalo, à la fin du XVI ${ }^{\mathrm{e}}$ siècle, circulaient du vin et de l'huile d'olive provenant d'Andalousie, ainsi que des produits d'outre-mer, tels que cochenille, bois brésil, clou de girofle, muscade, cannelle, gingembre, sucre, cuirs, sumac, etc. que les navires malouins rapportaient de Séville et de Lisbonne principalement $^{38}$.

Devant l'élargissement des marchés et l'abondance d'argent, le système des firmes basé sur les foires de change et le crédit dut lutter contre de nouvelles organisations qui réussirent à contrôler bonne part des échanges. La croissance des arrivées de métaux précieux se convertit en une opportunité pour mettre en place un système plus matériel dans lequel les nouveaux acteurs créèrent un circuit argent-marchandise-argent qui rivalisait avec celui qui prévalait en période de rareté de liquidité, le circuit créditmarchandise-crédit, ce dernier ayant subi de plein fouet les faillites sévillanes des années 1560-1570 et sur d'autres places de change européennes les années suivantes ${ }^{39}$. Car la multiplication relativement soudaine des acteurs et le développement des échanges entraînaient une certaine crise de confiance.

La proximité de Vitré mise à part, le fondement de cette grande activité maritime et commerciale était la fonction d'armement du port, affermie très tôt, dès la fin du XVI ${ }^{\mathrm{e}}$ siècle et le début du siècle suivant au moins. Les navires malouins étaient des frégates de 200 à 400 tonneaux construites dans le port et ses environs, qui étaient le plus souvent bien armées. En 1630, cette flotte atteignait 40 grandes unités, environ 11000/14000 ton-

y en León no vale tanto ni lo uno ni lo otro ». C23-227 Julien Ruiz à Simón Ruiz 23-4-1574 : "los reales son a 5\$". C27-223 Julien Ruiz à Simón Ruiz, 4-3-1575: "El real se cambia $5 \$ 3$ dineros ». C38-198 Julien Ruiz à Simón Ruiz 4-8-1577 : "Se gana mucho en mandar reales a Nantes: vale a 8 \$ el real y dicen que en Poitou a 9\$ ". C38-199 Julien Ruiz à Simón Ruiz, 2 septembre 1577 : "El real pasa a 12\$ entre mercaderes de Laval, Vitré, Beaufort."

36. GENTIL DA SiLVA, José, Lettres de Lisbonne, t. II, lettre n 250, p. 316. Même chose en 1578, lettre 265, p. 332. En 1577, grâce aux Français les réaux sont à 10 \% et vaudront plus de $20 \%$ à l'avenir.

37. En 1628, les réaux valait 6 sous en France, ce qui signifiait un gain confortable, SPOONER, Franck, op. cit., p. 194.

38. Du bois brésil, dès les années 1550, Arch. dép. d'Ille-et-Vilaine (Rennes), C 2877 : registre de recettes pour Saint-Malo, 1558-1559, marchandises sortantes; Archives municipales de Saint-Malo, CC 18-19 (1592-1593).

39. Pendant les années 1580, de nombreuses faillites ont lieu en Italie, à Anvers et à Lyon, LAPEYRE, Henri, Une famille..., op. cit, p. 453 sqq. 
neaux, outre les petites embarcations, ce qui représente un tonnage supérieur à celui des années $1660^{40}$. La zone entre l'île de Bréhat et Cancale offrait à l'économie maritime locale 400 marins, 500 charpentiers, 200 cannoniers et 50 bons maîtres ${ }^{41}$. Cette activité d'armement convertissait dès lors Saint-Malo en un port spécifique, hautement stratégique, en particulier pour le gouvernement français. Pour le roi et son royaume, l'intérêt de Saint-Malo et de ses marchands était double : ils permettaient de rapporter d'Espagne de l'argent en barres et en monnaie et ils représentaient un des potentiels navals les plus importants de l'Occident français.

Ces deux facettes de la puissance navale maritime malouine se reflètent dans les déclarations des ambassadeurs qui laissaient deviner l'avantage comparatif de Saint-Malo vis-à-vis des autres ports. Selon l'ambassadeur français, l'or et l'argent qui arrivaient à Séville n'y resteraient pas longtemps, car " [...] les marchands de bleds en tireront ceste année grande quantité, et ceulx qui y apportent aussi des toilles [... ${ }^{42}$ ". Plus tard, à l'occasion d'une négociation avec les autorités castillanes relative au séquestre de navires malouins, l'ambassadeur l'écrivait de façon encore plus claire : "J'y ay employé tout ce que j'ay peu, et en ay parlé fort franchement comme le faict le requeroit, car celluy des Bretons, importoit de tout le commerce $[\ldots]^{43}$."

Le rôle de pourvoyeur de métaux précieux que jouaient les Malouins et les autres Bretons disait beaucoup de l'intensité de leur commerce. L'hôtel des monnaies de Rennes, alimenté par les arrivées de métaux précieux à Saint-Malo, eut son premier apogée dans les années 1580, avec des totaux annuels aussi importants que ceux obtenus dans la seconde moitié du XVII ${ }^{\mathrm{e}}$ siècle $^{44}$. Cependant, il n'existait pas de corrélation stricte entre les arrivées de métal à Saint-Malo et les frappes monétaires à Rennes. En 1591, par exemple, arrivèrent seulement à l'hôtel des monnaies de Rennes 100000 livres d'argent (soit environ 33000 écus) alors que 500000 écus au moins parvinrent au port breton cette année-là. Les hauts et les bas

40. Correspondance de Henri d'Escoubleau de Sourdis : augmentée des ordres, instructions et lettres de Louis XIII et du Cardinal de Richelieu concernant les opérations des flottes françaises de 1636 à 1642, Paris, Imprimerie de Crapelet, 1839, vol. 3, p. 206. Selon l'inventaire de 1664, la flotte malouine était de 10000 tonneaux et de 20000 et 200 navires à la fin du XVIII ${ }^{\mathrm{e}}$ siècle; elle occupait à cette dernière période la quatrième ou conquième place au niveau national, BotTin, Jacques, BuTI, Gilbert et LeSPAGNOL, André, "Les moyens de l'échange maritime " dans Cabantous, Alain, Lespagnol, André et PÉron, Françoise (dir.), Les Français, la terre et la mer, XIII -XX $X^{e}$ siècle, Paris, Fayard, 2005, p. 271, 337.

41. Correspondance de Henri d'Escoubleau de Sourdis..., op. cit., p. 208.

42. Mousset, Albert, Dépêches diplomatiques de M. de Longlée (1582-1590), Paris, Librairie Plon, 1912, lettre envoyée à Villeroi, 10 août 1584, p. 106.

43. Ibidem, lettre de Longlée à Villeroi, 29 juin 1586, p. 279.

44. La comparaison avec les chiffres du XVII ${ }^{\mathrm{e}}$ siècle est intéressante, plus encore si l'on prend en compte la dévaluation de la livre entre le dernier tiers du XVI ${ }^{\mathrm{e}}$ siècle et la seconde moitié du XVII ${ }^{\text {e }}$ Les apports des Malouins et autres Bretons du Nord à l'hôtel des monnaies de Rennes furent aussi substantielles dans une période comme dans l'autre, SPOONER, Franck, op. cit. 
du total des frappes à Rennes étaient tout autant fonction du prix d'achat de l'argent américain par le gouvernement que du volume des arrivées. Manifestement, le tarissement du flux d'argent destiné aux frappes monétaires et que Franck Spooner observe pour la période postérieure à 1589 masque une partie des arrivées ${ }^{45}$. Le commerce continua à être actif et les Malouins vendirent leurs réaux et leurs barres d'argent au plus offrant ${ }^{46}$. Le prix supérieur qu'en proposaient les étrangers, surtout anglais et hollandais, interdisaient souvent aux officiers royaux de participer au négoce de l'argent, ce qui explique en bonne part le bas rendement de l'hôtel des monnaies rennais entre la fin du XVI ${ }^{\mathrm{e}}$ et les années $1640^{47}$. Cette baisse des frappes ne signifiait donc pas la fin d'échanges fructueux entre Saint-Malo (et les autres ports et villes bretonnes) et les places commerciales espagnoles ${ }^{48}$. Moins encore si l'on prend en considération la concurrence des officiers royaux de Saint-Lô, Angers et Tours qui se rendaient à Saint-Malo pour s'approvisionner en réaux. Au début de 1611, alors que le prix des réaux augmentait, même les agents de Rouen utilisaient des pièces venant de Saint-Malo ${ }^{49}$. Et le mois suivant, les habitants de Saint-Malo demandèrent l'autorisation d'envoyer des barres d'argent aux hôtels de monnaie qui leur offraient le plus d'avantages. Arguments qui montrent dans cet autre contexte que le commerce entre Saint-Malo et l'Espagne continuait avec vigueur dans la première moitié du XVII ${ }^{\mathrm{e}}$ siècle ${ }^{50}$.

Il ne fait pas de doute qu'il y a eu dans les ports du nord de la Bretagne, entre les années 1570 et 1630 un grand développement commercial. Si Rouen était bien le premier port du royaume, pour le commerce avec

45. Les centres bretons de production continuèrent d'exporter vers l'Espagne et l'Amérique dans la première moitié du XVII ${ }^{\mathrm{e}}$ siècle. En 1628, l'ambassadeur de France estimait à 5 millions d'écus, environ 15 millions de livres, la valeur des textiles français qui entraient principalement par Sanlúcar, Cadix et Séville.

46. À la fin du XVI ${ }^{\mathrm{e}}$ siècle, les Malouins refusèrent l'implantation d'un hôtel des monnaies à Saint-Malo, affirmant qu'ils voulaient vendre leurs réaux au plus offrant. En 1594, Flamands et Hollandais négociaient en Bretagne l'achat de monnaies d'argent, LESPAGNOL, André, op. cit., p. 151, 476-478; LE Goff, Hervé, La Ligue...., op. cit. ; SPOONER, Franck, op. cit., note 1 p. $175-176,179,184$.

47. Durant le règne de Henri IV, le commerce du blé s'élevait à 4 millions d'écus d'or, soit environ 12 millions de livres, ce qui situerait le blé immédiatement après les textiles dans les exportations françaises vers l'Espagne, GIRARD, Albert, op. cit., p. 347.

48. En 1607, on parlait aussi de la possibilité d'un hôtel des monnaies à Morlaix, car les commerçants de la ville possédaient des barres d'argent, fruit de leur commerce textile et céréalier en Espagne. À cette époque, ils alimentaient l'hôtel des monnaies de Rennes, tandis que Saint-Malo approvisionnait Saint-Lô, Rouen et d'autres ports, SPoonER, Franck, op. cit., p. 185.

49. Ibidem.

50. En 1596, un agent anglais affirmait qu'à Morlaix, il y avait des marchands qui avaient de grosses affaires à Sanlúcar, LE Goff, Hervé, op. cit., p. 409 note 225. Une instruction de 1654 expliquait que " las naos francesas de Saint-Malo y Rouen, cada una con 1500 fardos, iban a ponerse a veinte leguas de la frontera en Cádiz, en donde con los que navegaban en la carrera de Indias ajustaban sus tratos y comercio, y también los introducían por todos los demás puertos y los pasaban a las partes de Castilla ", cité par AllozA, Ángel, op. cit., note 41, p. 95. 
l'Espagne le port normand dut partager sa prééminence avec ses voisins bretons de Saint-Malo, Vitré et Morlaix, en particulier.

\section{Modalités de la protection des biens et de l'installation des Malouins en terre étrangère}

La grande variété des activités malouines et ses connexions avec le pouvoir politique français (à travers la course et l'approvisionnement en argent-métal) étaient liées à son pouvoir guerrier. Armes et argent rendaient ces acteurs indispensables au bon fonctionnement de l'économie française et espagnole, et à la défense du territoire des deux monarques. Ils constituaient des collaborateurs nécessaires et jusqu'à un certain point indispensables, et étaient par conséquent craints des gouvernements pour leur indépendance.

En 1585, l'ambassadeur français à Madrid mentionnait qu'un navire de Saint-Malo appelé " Le Croissant ", de 400 tonneaux de jauge, avait subi un embargo à Sanlúcar, à la demande du roi de Castille, au moment où il retournait en Bretagne avec 120000 écus à son bord ${ }^{51}$. Ce navire, cédé à des particuliers pour la carrera de Indias - car il était grand et très bon précise le texte - avait été vendu contre l'avis du conseil des Indes qui demandait que le navire soit rendu à ses propriétaires moyennant le paiement d'une caution. Grâce à des lettres postérieures, nous savons que ce navire s'était rendu en Espagne avec d'autres bateaux bretons et qu'ils devaient repartir vers la Bretagne avec plus de 300000 écus en argent et en marchandises. Les exportations de métaux précieux vers l'étranger étant interdites, vingt marchands bretons avaient été emprisonnés, mais le navire avait finalement été rendu à ses propriétaires ${ }^{52}$.

Les raisons du succès de cette médiation de l'ambassadeur devaient beaucoup à l'importance commerciale des Malouins en Andalousie, mais aussi à l'importance de leurs armes car, lorsque leurs navires et leurs réaux ne leur étaient pas restitués, les Malouins «[...] fond[ai]ent incontinent sur des lettres de marques et représailles [...] ", ce qui ne laissait pas d'inquiéter le roi castillan ${ }^{53}$. Lors du séquestre du navire breton en Andalousie, une lettre de représailles avait été donnée au Malouin Jean Le Breton pour le navire "Grand Guy " afin d'armer en course contre les Espagnols ${ }^{54}$. Elle avait été révoquée dès que le secrétaire d'État et de guerre, Juan de Idiáquez, avait promis de satisfaire les parties demandantes. La menace des armes de ceux qui avaient été dépouillés leur permettait de récupérer leurs

51. Plusieurs navires et marchandises de Bretons avaient subi un embargo cette annéelà, et Longlée avait été sollicité par leurs propriétaires pour réclamer la restitution de leurs biens devant le conseil, Mousset Albert, Dépêches diplomatiques..., op. cit., lettres à Henri, 14 avril 1585, 20 février 1586, p. 128-129; 229-230.

52. Ibidem, Longlée à Villeroi, 29 juin 1586, p. 279 et Longlée à Henri III, 15 mars 1588, p. 358 .

53. Longlée à Henri III, 14 avril 1585, p. 128-129.

54. Longlée à Villeroi, 29 juillet 1584, p. 163. 
biens, mais tous les marchands ne pouvaient pas se prévaloir de cette capacité offensive, les ports d'armement ayant en la matière un atout de choix.

Comme je l'ai signalé plus haut, le rôle des armes allait plus loin. En de nombreuses occasions, le roi castillan requérait également le pouvoir maritime et militaire des Malouins. Certains d'entre eux réalisaient des activités d'espionage pour le compte du roi castillan ${ }^{55}$ tandis que d'autres participaient à la lutte contre les ennemis de l'Espagne en Amérique, comme en 1624, lorsque trois navires de Saint-Malo et leur équipage avaient été incorporés dans une escadre qui allait au Brésil donner la chasse aux Hollandais ${ }^{56}$. Pour éviter toute contestation, quatre mois de fret et de salaires avaient été payés à l'équipage et aux propriétaires, ce qui suppose par ailleurs que ces gens de mer et de guerre connaissaient bien les itinéraires transatlantiques ${ }^{57}$. Même après 1635, en période de guerre ouverte entre Français et Espagnols, les Malouins savaient se montrer généreux avec les Espagnols ${ }^{58}$.

La relation entre les Malouins et le pouvoir royal qu'il soit français ou espagnol était fréquente sinon continue : les Bretons approvisionnaient en armes certains ports espagnols, comme celui de Carthagène et un représentant de la famille Magon, famille bien connue en Bretagne, était pourvoyeur de la carrera de Indias à Cadix ${ }^{59}$. De plus, les Malouins figuraient aux avant-postes du commerce français et comme intermédiaires privilégiés avec les autorités espagnoles : le 7 novembre 1659, le négociant malouin Guillaume Éon de Villegille (1611-1671), était nommé consul français de Cadix et l'année suivante, parmi les six consuls nommés pour gérer l'ensemble des postes consulaires dans la Péninsule ibérique, apparaissaient le sieur Trouin qui s'occupait de Gibraltar, Carthagène et Malaga et Guillaume

55. Archivo General de Indias-Filipinas 200, ${ }^{\circ}$ 177. Déclaration de Michel Colonel, originaire de Saint-Malo, capitaine du navire "L'Espérance " qui entra à Sanlúcar en 1617, sur son séjour à Plymouth, port duquel il était sorti 18 jours auparavant après y avoir résidé six semaines. Il affirmait que dans la Tamise on armait et avitaillait 10 ou 12 embarcations avec force artillerie et gens de guerre sous le commandement de Walter Raleigh.

56. GIRARD Albert, El comercio..., op. cit., p. 90. Il est question de cinq navires de Saint-Malo et de près de 400 marins dans le Mercure François (1625), p. 495, cité dans Hugon Alain, Au service du roi catholique, honorables ambassadeurs et divins espions. Représentation diplomatique et service secret dans les relations hispano-françaises de 1598 à 1635, Madrid, Casa de Velázquez, 2004, p. 246.

57. Des Malouins avaient participé à l'expédition de Magellan en 1519, LE Goff Hervé, op. cit., p. 28.

58. En 1636, un autre Malouin, La Chesnaye, avec le Griffon de 200 tonneaux, avait capturé une grande caravelle turque (de Salé) avec 40 Espagnols que le Malouin avait libérés à Gibraltar malgré la guerre ouverte entre Français et Espagnols à cette date, Correspondance de Henri d'Escoubleau de Sourdis : augmentée des ordres, instructions, et lettres de Louis XIII et du Cardinal de Richelieu a M. de Sourdis concernant les opérations des flottes françaises..., 1839, vol. I, p. 47.

59. Quand Philippe II portait son concours financier et militaire au duc de Mercœur à la fin des années 1590, on sollicita le marchand malouin, Julien Gravé, pour obtenir de la poudre, Gaston de CARNÉ, Documents sur la Ligue en Bretagne. Correspondance du duc de Mercoeur et des ligueurs bretons avec l'Espagne, Vannes-Rennes, 1899, t.1, doc. 19, p. 14. 
Éon Villegile qui fut établi pour les places de Cadix, Port-Sainte-Marie, San Lucar de Barrameda et Séville ${ }^{60}$.

Ces faits, loin d'être anecdotiques (voir également le texte de Vicente Montojo), correspondaient à la spécificité malouine, à la fois marchands de mer et guerriers, soucieux de jouer les premiers rôles face aux autorités du pays d'accueil, spécificité en bonne part responsable du succès de leurs affaires dans un contexte d'arrivées abondantes de métaux précieux et de tension belliqueuse maximale, entre les puissances du nord-ouest européen et l'Espagne.

Observer les agissements de Bretons du Nord en Andalousie nous amène à prendre en compte le processus de sédentarisation de certains d'entre eux en terre hispanique. Si jusque vers 1580, ils étaient majoritairement itinérants, réalisant des va-et-vient entre les ports de Séville, Cadix, Sanlúcar, Santa María et celui de Saint-Malo, sans s'établir durablement dans ces ports ${ }^{61}$, par la suite, ils s'installèrent plus souvent en terre andalouse. Ils y diversifièrent leurs négoces, y investirent tant dans le commerce de gros que de détail et, via des lettres de naturalité ou de façon illégale, certains devinrent même cargadores aux Indes.

Il est évident que pour diversifier leurs affaires et s'assurer de meilleurs débouchés en Espagne et en Amérique, les Bretons du Nord devaient pouvoir compter sur des relais en Andalousie. Une gestion partagée des affaires entre la Bretagne et l'Espagne permettaient aussi aux Malouins et aux Vitréens qui restaient en France de pouvoir investir simultanément dans d'autres négoces sans avoir à voyager à bord des frégates accompagnant la marchandise jusqu'à Sanlúcar ou Cadix. Une fois le négoce affermi, leur présence en Andalousie offrait l'opportunité de vendre à crédit et d'occuper le marché de façon croissante. En termes généraux, à travers la création d'un nouveau pôle d'activité, le négoce malouin et vitréen se renforçait.

Si les membres des familles établies en Andalousie ne participèrent pas tous avec la même intensité au commerce andalou, il semble tout aussi important de remarquer qu'elles étaient reliées entre elles et avaient des activités complémentaires. En Andalousie, les Bretons du Nord remplissaient différentes fonctions : tandis que nombre d'entre eux étaient grossistes ou marchands de détail, d'autres se convertissaient en plateros - office étroitement lié au travail de l'or et de l'argent - ou en pasajeros vers l'Amérique. L'office d'orfèvre était particulièrement intéressant car il

60. Nous remercions Sylvain Lloret de ces informations qui figurent dans sa thèse de doctorat, dont la soutenance s'est tenue récemment.

61. Cela n'empêche nullement que certaines familles aient pu s'établir antérieurement dans ces ports, dès le Moyen Âge, MÉnANTEAU, Loïc, "Sanlúcar de Barrameda y Bretaña " dans Rubiales Torrejón, Javier, El río Guadalquivir, vol. 2, Séville, Junta de Andalucía, 2011, p. 186-189. Je remercie José Ignacio Martínez Ruiz d'avoir attiré mon attention sur ce travail. 
permettait l'échange des monnaies d'or et de celles d'argent en fonction de leur valeur en France ${ }^{62}$.

L'éventail des occupations possibles comprenait aussi des fonctions officielles ou officieuses dans la carrera de Indias (informateurs, chirurgiens et pourvoyeurs) et au service du monarque castillan, comme nous l'avons vu. C'est dire que ces marchands avaient réussi à offrir une longue liste de services au roi et au royaume où commerce de marchandises, argent, transport, espionnage et guerre interagissaient. Grâce à leur mobilité, aux services rendus au roi ennemi de la France, ils étaient parvenus à brouiller les frontières politiques et les antagonismes, et à faire en sorte que leur fût réservé un traitement préférentiel dans les deux royaumes.

Parmi les grandes figures politiques et économiques malouines en Andalousie, l'on trouve Alberto Juan (Albert Jean Tréhouart), Sebastián Trehuarte (Sébastien Tréhouart) et Alonso Magon (Allais ou Alain Magon) ${ }^{63}$. Les deux premiers furent naturalisés, Alberto Juan en 1624 et Sebastián dix ans plus tard. Nous avons quelques informations concernant l'envergure commerciale d'Alberto Juan en Andalousie, grâce à l'embargo de 1635 durant lequel on lui séquestra 85000 ducats environ, la moitié en argentmétal $^{64}$. Quant à Sebastián Trehuarte, " naturel " de Saint-Malo, sieur de la Gastinais et vecino de Séville ${ }^{65}$, il apparaît comme pasajero à destination de l'Amérique dans la flotte de 1634. Il était associé à un Rouennais, Pedro de Fuentes, avec lequel il s'était rendu à Veracruz, et avec Diego de Torres Castroverde et Mateo de Castroverde ${ }^{66}$. Le Malouin faisait aussi affaire avec Pedro de la Farxa, un des plus grands et plus riches marchands français d'Andalousie ${ }^{67}$.

62. En 1625, Pierre Hamon, orfèvre à Cadix, est originaire de Saint-Malo, Archivo Histórico Provincial de Cádiz, SM CA 3030 107-109. À la même époque, Jean Hévin, originaire de Vitré et lié au milieu malouin est platero de oro à Séville, FRAIN DE LA GAULAYRIE, Édouard, "Commerce des Vitréens en Espagne, 1629-1630 », Vitré, Imprimeur E. Lécuyer, 1898, p. 9, 11, 17.

63. Alberto Juan était arrivé à Séville en 1596. Cet Alberto Juan était un membre de la famille Tréhouart, voir CRAILSHEIM, Eberhard, " Les marchands français à Séville (15801650). Les exemples de Pedro de la Farxa, Lanfran David et Pedro de Alogue " dans PRIOTTI, Jean-Philippe et SAUPIN, Guy (dir.), Le commerce atlantique franco-espagnol (XV. XVIII siècles). Acteurs, négoces et ports, Presses Universitaires de Rennes, 2008. Un Alain Magon de Saint-Malo s'était marié avec Jeanne Moreau en 1600. Nicolas Magon (16041661), son fils, se maria, pour sa part, avec Perrine Grout en 1633 et ils laissèrent un héritage de 300000 livres. Un autre Alain (1601-1683), aîné du couple Magon/Moreau (mentionné dans nos sources), fonda sa propre maison de commerce à Cadix, ville dans laquelle il s'établit en 1615, LesPagnol, André, op. cit., t. I p. 126 et t. IIp. 843.

64. Alloza ApARICIO, Ángel, op. cit.,p. 91.

65. Archivo General de Indias-Contratación, 537, nº 2, r2 y contratación 5416 , n 9. Il nomma comme héritiers universels sa mère Gilette Ascoul, veuve d'Étienne Tréhouart, qui vivait et résidait à Saint-Malo et, à défaut, ses frères André, Thomas, Guillaume, Gilette et Laurent Tréhouart, à parts égales.

66. Archivo General de Indias-Contratación 5415, nº 111.

67. Il était débiteur, à moitié avec Fuentes, de Pedro de la Farxa, auvergnat de Séville de 66727 réaux d'argent, à payer au Mexique. Ils étaient aussi débiteurs à hauteur de 
En ce qui concerne la famille Magon, elle ocupa dans le port gaditain une place de choix. Un certain Guyon Magon s'était établi à Cadix au milieu du $\mathrm{XVI}^{\mathrm{e}}$ siècle et il s'était marié à la sœur d'un régidor de la ville. Et au début du $\mathrm{XVII}^{\mathrm{e}}$, un autre membre de la famille, Allais Magon, allait émigrer à Cadix et se convertir en un personnage de premier plan alliant commerce et fonction de pourvoyeur royal.

Par ailleurs, dans les années 1629-1630, à peu de distance de Cadix, de nombreux Vitréens commerçaient en gros à Sanlúcar, tels les frères Hévin qui avaient une compagnie en Bretagne avec leur père et d'autres associés, dont certains installés à Sait-Malo, et agissaient aussi pour le compte de parents et autres collaborateurs à Nantes, Rouen, Middelbourg, Rotterdam et Amsterdam ${ }^{68}$. À Séville, un de leurs cousins était orfèvre et, à travers un autre parent, ils faisaient le commerce avec l'Amérique. Ils commerçaient aussi bien du vin de Jerez, de l'eau-de-vie, des raisins secs, des citrons et des oranges d'Andalousie, du tabac, du gingembre et de la cochenille d'Amérique que des draps de Rouen, des toiles de Bretagne (" morlaix ", " crées "), des anascotes de Bruges, des "bramantes " - qui se fabriquaient à Gand, Bruges, Courtray et Ypres -, des fardeaux de " gantes ", des " cotonies " de Lyon, de la soie de Paris, etc., ce qui dévoile l'extension de leur zone d'approvisionnement notamment textile. Le mode de paiement qu'ils utilisaient ne se calquait pas nécessairement sur celui des Malouins et d'autres Vitréens du XVI ${ }^{\mathrm{e}}$ siècle. Pendant les sept premiers mois de 1630, compte tenu des risques de mer, ils utilisèrent des lettres de change dont les mouvements entre Séville d'un côté et la Bretagne, la Normandie et la Hollande de l'autre, représentaient quelque 15000 écus ${ }^{69}$.

Ces grandes familles maintenaient des relations avec d'autres Malouins et Vitréens établis en Andalousie et en Bretagne, de même qu'elles servaient de lien principal avec d'autres Français, auvergnats et rouennais présents en Andalousie. Elles chargeaient majoritairement leurs marchandises sur des navires malouins et à destination du port breton. Charles Baulier, originaire de Saint-Malo, était marchand à Cadix et pourvoyeur des boutiques et des marchands-ambulants de la ville ${ }^{70}$. Célibataire de 25 ans, il habitait dans la calle vestuario de las comedias, et son frère cadet l'aidait dans sa tâche, tandis que Mathurin Le Moyne était son associé ${ }^{71}$. Parmi ses débiteurs apparaissaient d'autres Malouins comme François Gouverneur,

15700 réaux de Francisco Campi, de Séville, qu'ils devaient rembourser à Veracruz à son frère, Rafael Campi.

68. Voir la correspondance des frères Hévin analysée par FRAIN DE LA GAULAYRIE, Édouard, " Commerce des Vitréens en Espagne, 1629-1630 », Vitré, Imprimeur E. Lécuyer, 1898, p. $1-29$.

69. Ibidem, p. 13 note 2. Même s'ils recouraient habituellement aux Malouins, ils pouvaient aussi confier leurs biens à des marins et charger sur des bateaux d'Olonne, Le Croisic, Nantes, Dieppe et Le Havre qui faisaient le voyage de Sanlúcar en Bretagne.

70. Archivo Histórico Provincial de Cádiz, inventario nº 866. Fils de Juan Bollier et de Francisca Buduart. Le père était mort et la mère vivait à Saint-Malo.

71. Baturin Lemuena (Le Moyne) lui devait de l'argent de comptes communs. 
ou des Français comme Bernard Bovin qui tenait boutique dans la calle de flamencos ${ }^{72}$. À sa mort, en 1647, il avait 13000 réaux de créances, et pas de dettes. Aux mêmes dates, Alonso de la Haya, d'une famille bien connue à Saint-Malo, avait investi dans de nombreux négoces (ventes et achats de sucre, change d'argent et d'or) et avait des liens commerciaux avec Juan Trublet et Juan Gallardo Picote, Malouins comme lui ${ }^{73}$.

Dans d'autres cas, la nécessité d'être entouré de compatriotes n'est pas immédiatement perceptible. Il ne semble pas que Miguel Compon maintînt de nombreux contacts avec des ressortissants malouins établis en Andalousie. Il était à Cadix depuis 1615 au moins et on le voyait essentiellement lié aux Portugais, parmi lesquels apparaissaient ses associés de Puerto Santa María, ses parents et, en partie, ses débiteurs ${ }^{74}$. Cependant, le type de marchandises qu'il vendait renvoyait au port de Saint-Malo et au reste de la Bretagne. Dans un état de comptes des années 1616-1625, il avait à son actif des créances pour du blé vendu, pour des textiles sortis de sa boutique, des prêts aussi, pour un total d'environ 1600 ducats. Son activité dépassait les limites du port puisqu'en 1624 il administrait la dîme de la banda mayor del pan de la ville de Lebrija ${ }^{75}$.

Ces exemples qui montrent la fréquence de l'établissement des Malouins sur la côte andalouse et la variété de leurs négoces ne doivent pas nous faire oublier qu'une grande partie de ce commerce était toujours réalisée par des acteurs itinérants faisant le voyage entre le nord de la Bretagne et la côte andalouse. C'est pour cette raison qu'une étude de leur présence en Andalousie ne dit pas tout. La preuve en est que s'ils gênèrent dans une certaine mesure le commerce breton, ni la course, ni les représailles, ni les officiers chargés de la contrebande, en finirent réellement avec ce commerce. De part et d'autre de 1635, le flux de métaux précieux continua à circuler entre la baie de Cadix et Saint-Malo (voir annexe 1) ${ }^{76}$.

Il ne fait pas de doute que les flux de métaux précieux donnèrent lieu à de nouveaux circuits commerciaux et favorisèrent l'enrichissement de nouveaux protagonistes, parmi eux, ceux de Saint-Malo et de Vitré. Malgré sa supériorité au niveau général, le port de Rouen était loin de contrôler tout le commerce atlantique avec l'Andalousie. La possession d'une flotte

72. Francisco Governador (Gouverneur) devait 400 réaux à Bollier que ce dernier lui avait prêté pour sa boutique, Archivo Histórico Provincial de Cádiz, inventario nº 871.

73. Archivo Histórico Provincial de Cádiz, numéros 688 et 891.

74. Archivo Histórico Provincial de Cádiz, numéro 900.

75. Pour 350 ducats. Cette année-là, avaient été reçus 3400 fanègues, 800 d'orge et le reste de blé. Bien que ses derrnières volontés semblent marquer un certain éloignement du milieu marchand malouin et français, il continuait sans doute à faire du commerce avec la Bretagne, zone céréalière importante à cette époque.

76. Alloza ApARICIO, Ángel, op. cit., p. 138, 142. Par exemple, entre juillet 1636 et octobre 1638, le marchand anglais Benjamin Ruit obtint une licence pour pouvoir faire entrer en Espagne 950000 ducats de marchandises, matériaux et équipements divers, à condition qu'ils soient transportés sur des navires français. Entre 1636 et 1640, on accorda des autorisations spéciales pour introduire 4,5 millions de ducats de marchandises françaises. 
était en la matière un avantage de première importance, avantage dont Rouen ne jouissait pas. De cette façon, les Malouins avaient pu rationaliser les circuits commerciaux boycottant le rôle d'intermédiaires joué par les Espagnols et intégrant les activités de production-transport-commercialisation. Grâce à la détention de la force armée - et en échange de services militaires et diplomatiques - ils avaient réussi à se ménager le soutien des deux monarques les plus puissants du moment qui luttaient pour la suprématie européenne. En Andalousie (Cadix et Sanlúcar principalement), ils avaient contribué à créer de nouvelles zones de commerce hors de portée des officiers royaux, commerçant avec du comptant de port en port. De cette façon, ils avaient abaissé leurs coûts de transaction réduisant ainsi les problèmes liés à la confiance. Une fois leurs affaires affermies, ils s'étaient plus fréquemment installés en Andalousie, diversifiant leurs activités et, selon les circonstances, leur mode opérationnel.

Il est symptomatique que - de l'autre côté de l'Atlantique -, les Peruleros aient eu à la même époque une organisation similaire. En effet, l'augmentation de la production d'argent et de son exportation vers l'Andalousie promut d'autres processus concurrentiels, entre Peruleros et Sévillans, cette fois. Certains acteurs provenant du Pérou - bien que nombre d'entre eux aient été fils d'Espagnols - commencèrent à contester le monopole sévillan et à contrôler une bonne part des échanges transatlantiques. Le paiement au comptant et le boycott des foires de Panama furent aussi à l'origine de leur succès en Andalousie ${ }^{77}$. À l'image des Malouins, les atouts des Peruleros étaient la mobilité et l'argent sonnant avec lequel ils arrivaient à Séville. Ils voyageaient avec des barres d'argent jusqu'en Andalousie et achetaient au comptant des toiles françaises, non seulement en Andalousie mais aussi en Biscaye, autre zone qui fournissait des toiles. Peruleros et Malouins devaient finir par faire affaire ensemble, malgré les plaintes du consulat sévillan.

En définitive, en développant le potentiel d'échanges, les arrivées massives d'argent provoquèrent la promotion de nouveaux centres commerciaux et une nouvelle hiérarchie de ces centres, une certaine restructuration des circuits commerciaux et des marchés ainsi que l'émergence de nouveaux groupes d'entrepreneurs. La concurrence et les coûts de transaction jouaient un rôle fondamental dans cette dynamique, aussi bien dans les modifications de l'organisation des firmes et dans la répartition des différentes marchandises sur les différents marchés, que dans la géographie du commerce et de la répartition des fonctions marchandes. Aussi, il convient de relativiser l'opposition entre les théories d'Adam Smith et de Douglass

77. Au sujet de la simultanéité des processus concurrentiels entre espagnols, américains et étrangers, je me permets de renvoyer à mes travaux, "Una conquista "al revés" o la penetración de los mercaderes noreuropeos y americanos en la Península Ibérica (1560-1630) " dans Mazín Gómez, Óscar (dir.), México y el mundo hispánico, México, El Colegio de Michoacán, 2000, p. 207-30; « Metales preciosos... »; « Logiques commerciales d'une globalisation ". 
North, car le rôle important des coûts de transaction, l'agrandissement et la spécialisation des marchés semblent avoir été compatibles ${ }^{78}$.

C'est précisément à travers l'apparition de nouveaux groupes d'acteurs - qui grâce à leur propre organisation et à leur logique commerciale arrivaient à abaisser les coûts de transaction - que s'effectuèrent les changements liés à la spécialisation des marchés. Il faut noter cependant que la variation de ces coûts dépendait tout autant des rivalités entre les différents groupes de marchands, de la relation de chacun d'eux à son environnement et d'une conception particulière des affaires, qu'à un cadre institutionnel imposé par l'État. En d'autres termes, l'économie - ici le commerce - était aussi régie par des processus autonomes, c'est-à-dire que les agissements des groupes de marchands " nationaux " doivent non seulement être entendus en fonction des relations maintenues avec le pouvoir politique mais aussi, dans un contexte de concurrence internationale, dans le champ économique même.

\section{Annexe 1 - Argent à destination de Saint-Malo en provenance d'Andalousie ${ }^{79}$}

\begin{tabular}{|c|c|c|c|}
\hline Date & $\begin{array}{c}\text { Quantités exportées } \\
\text { illégalement }\end{array}$ & Nombre de navires & $\begin{array}{c}\text { Frappes monétaires à } \\
\text { Rennes } \\
\text { Argent/or (en livres) }\end{array}$ \\
\hline 1578 & & & $635750 / 8231$ \\
\hline 1579 & & & $719882 / 4579$ \\
\hline 1580 & & & $946455 / 2593$ \\
\hline 1581 & & & 1356566 \\
\hline 1582 & & & 1566980 \\
\hline 1583 & & & $788376 / 10249$ \\
\hline 1584 & & Dans plusieurs navires & $627990 / 3060$ \\
\hline 1585 & $\begin{array}{c}300000 \text { écus d'argent } \\
\text { et des marchandises }\end{array}$ & & $453339 / 3686$ \\
\hline 1586 & & & \multicolumn{2}{|c}{} \\
\hline
\end{tabular}

78. Pour Smith, l'expansion européenne sur le monde et, partant, le développement des échanges avaient provoqué la spécialisation des marchés, Smith, Adam, La Richesse des nations, Paris, Flammarion Poche, 1999 ( $1^{\text {re }}$ éd. en anglais en 1776), t. I tandis que Douglass North insistait sur la primauté explicative des coûts de transaction, principal agent du changement institutionnel et de l'efficience économique, NORTH, Douglass C., Institutions, Institutional Change and Economic Performance, Cambridge, Cambridge University Press, 1990.

79. Sources : FRAIN DE LA GAULAYRIE, Édouard, Les Vitréens et le commerce international..., op. cit., p. 48; Mousset, Albert, Dépêches diplomatiques de M. de Longlée..., op. cit.; "Correspondance de Jean Penninck, greffier de l'Amirauté de Dunkerque (1631-1646) ", publiée entre 1984 et 2003 dans la Revue de la société dunkerquoise d'Histoire et d'Archéologie; SPOONER, Franck, op. cit.,p. 407-409. 
Jean-Philippe PRIOTTI

\begin{tabular}{|c|c|c|c|}
\hline Date & $\begin{array}{c}\text { Quantités exportées } \\
\text { illégalement }\end{array}$ & Nombre de navires & $\begin{array}{c}\text { Frappes monétaires à } \\
\text { Rennes } \\
\text { Argent/or (en livres) }\end{array}$ \\
\hline 1587 & & & $1621955 / 16859$ \\
\hline 1588 & & & $1146417 / 63523$ \\
\hline 1589 & & & $262508 / 14001$ \\
\hline 1591 & 500000 écus d'argent $^{a}$ & Dans deux navires & $100794 / 4321$ \\
\hline 1592 & & & $48422 / 10462$ \\
\hline 1593 & & & $55717 / 6307$ \\
\hline 1594 & & & $89019 / 1305$ \\
\hline $1595^{*}$ & & & $109507 / 2831$ \\
\hline $1596^{*}$ & & & $138110 / 3261$ \\
\hline $1597^{*}$ & & & $55072 / 2610$ \\
\hline $1598^{*}$ & & & 27992 \\
\hline 1599 & & & $71696 / 3342$ \\
\hline 1600 & & & $97859 / 4567$ \\
\hline 1601 & & & $94470 / 2391$ \\
\hline 1602 & & & $246938 / 3169$ \\
\hline 1603 & & & $462672 / 2356$ \\
\hline 1604 & & & 254016 \\
\hline 1605 & & & 462914 \\
\hline 1606 & & & 197770 \\
\hline 1607 & & & 215551 \\
\hline 1608 & & & 151603 \\
\hline 1609 & & & 69310 \\
\hline 1610 & & & 87454 \\
\hline 1611 & & & 93623 \\
\hline 1612 & 250000 écus d'or & Dans deux navires & 86365 \\
\hline 1613 & & & 86184 \\
\hline 1614 & & & 39372 \\
\hline 1615 & & & $206307 / 10125$ \\
\hline 1616 & & & 166925 \\
\hline 1617 & & & 113809 \\
\hline 1618 & & & 80418 \\
\hline 1619 & & & 26179 \\
\hline 1620 & & & 19958 \\
\hline 1621 & & & 7893 \\
\hline 1623 & & & 29615 \\
\hline
\end{tabular}


Au cœur des échanges européens

\begin{tabular}{|c|c|c|c|}
\hline Date & $\begin{array}{c}\text { Quantités exportées } \\
\text { illégalement }\end{array}$ & Nombre de navires & $\begin{array}{l}\text { Frappes monétaires à } \\
\text { Rennes } \\
\text { Argent/or (en livres) }\end{array}$ \\
\hline 1624 & & & 67677 \\
\hline 1625 & & & 13951 \\
\hline 1638 & $\begin{array}{c}44300 \text { florins, } 3 \text { barres } \\
\text { d'argent et } 16 \text { fardeaux } \\
\text { de toiles }\end{array}$ & $\begin{array}{c}\text { Dans un navire breton } \\
\text { de } 60 \text { lasts avec } 10 \\
\text { pièces de canon }\end{array}$ & \\
\hline 1642 & $\begin{array}{c}400000 \text { florins, plus des } \\
\text { lingots d'or, } 5183 \text { cuirs, } \\
\text { sacs de laine, coche- } \\
\text { nille, alun, etc. }\end{array}$ & Dans un navire & $111688 / 3276$ \\
\hline 1654 & 400000 couronnes $^{\mathrm{b}}$ & Dans 5 navires & \\
\hline 1657 & $\begin{array}{c}170000 \text { livres sterling } \\
\text { en argent et presque } \\
\text { autant en marchan- } \\
\text { dises }^{\mathrm{c}}\end{array}$ & Dans un navire & \\
\hline
\end{tabular}

* Sont incluses les frappes de Dinan.

a. Grégoire, Louis, La Ligue..., op. cit., p. 108, 253. En 1591, les navires de Saint-Malo de retour d'Espagne avec 500000 écus à leur bord, jetaient l'ancre à Blavet, port sous contrôle espagnol, à cause de la menace que représentaient les navires anglais en Bretagne.

b. A Collection of the State Papers of John Thurloe, Volume 2, 1654. Originally published by Fletcher Gyles, London, 1742.

c. A collection of the State Papers of John Thurloe, Volume 6, January 1657-March 1658. Originally published by Fletcher Gyles, London, 1742. General Mountagu to secretary Thurloe. Vol. LIX, p. 61. Aboard the Nasebye in the Downes, September 7, 1657. 
Annexe 2 - Malouins et Vitréens à Séville et à Puerto Santa María, 1606-1634 ${ }^{80}$

\begin{tabular}{|c|c|c|c|}
\hline Année & Nom & Domicile & Origine géographique \\
\hline $1606^{*}$ & Pierre Morel & Puerto de Santa María & Sans doute de Vitré \\
\hline $1611^{*}$ & Nicolas Gravé $^{\text {a }}$ & Sanlúcar/Séville & Saint-Malo \\
\hline $1624^{*}$ & Albert Jean Tréhouart & Saint-Malo \\
\hline 1625 & Jacques Trublet & $\begin{array}{c}\text { Marchand-grossiste à } \\
\text { Séville }\end{array}$ & Saint-Malo \\
\hline 1625 & Claude Danycan & $\begin{array}{c}\text { Marchand-grossiste à } \\
\text { Séville }\end{array}$ & Saint-Malo \\
\hline $1630^{*}$ & Allais Magon & Séville & Saint-Malo \\
\hline $1633^{*}$ & Étienne Trublet & Séville & Saint-Malo \\
\hline $1634^{*}$ & Sébastien Tréhouart & Séville & Saint-Malo \\
\hline
\end{tabular}

* Année d'obtention de la lettre de naturalité.

a. Nicolas Gravé était né à Sanlúcar, de parents malouins.

\section{Annexe 3-Marchands malouins actifs à Cádiz (1616-1648) ${ }^{81}$}

Étienne Trublet ${ }^{82}$

Jean Trublet (résident de Cadix)

Alain Magon, marchand-grossiste, pourvoyeur des flottes

François Pépin ${ }^{83}$

Alain de la Haya

Marc de la Haya

Pierre Hamon

Julien Hamon

80. À partir des données contenues dans CRAILSHEIM, Eberhard, « Les marchands français à Séville (1580-1650)... ", art. cit., tableau n ${ }^{\circ} 1$, que nous avons complétées, ajoutant parfois le lieu d'origine (cas d'Étienne Trublet et de Sébastien Tréhouart), adaptant parfois le patronyme à l'orthographe française (cas de Nicolas Grane que l'on doit comprendre comme Nicolas Gravé).

81. Archivo Histórico Provincial de Cádiz : Carlos Bollier CA 2513 101-103, Esteban Trublet CA 5509 1207-1210, Miguel Compon CA 4373 1-2, Miguel Compon CA 5147 111-128, Pedro Hamon CA 3030 107-109, Alonso de La Haya CA 2514 291-292, Guillermo Letura CA 2514 94-95, Fedrique Domenico CA 3031 276-279.

82. Les Trublet sont une des plus anciennes familles de la bourgeoisie malouine, LESPAGNOL, André, op. cit.

83. Les Pépin appartenaient à la noblesse " ducale " établie dès le milieu du Xve siècle à Saint-Malo, période durant laquelle ils mirent leur noblesse " en dormition " afin de pouvoir exercer le commerce maritime, LesPaGnol, André, op. cit. 
Michel Compon (résident de Cadix, habitant de Rota)

Guillaume Letura (résident de Cadix)

Charles Bollier

Jean Bollier

Jean Gaillard Picot (résident de Cadix)

Mathurin Le Moyne*

François Gouverneur

peut-être originaire de Vitré

\section{Annexe 4 -Marchandises chargées sur la flotte de la Nouvelle Espagne en 1634 par Sebastián Trehuarte et Pedro de Fuentes}

9 pièces de rouens : 333 aunes qui font 522 varas $3 / 4$

8 pièces de rouens blancarts : 349 aunes qui font 549 varas

130 varas de mélinges

42 demi-pièces d'olonnes

11 pièces de bramante : 340 varas

6 pièces d'étoupe

8 gros de ruban de gamusa

23 pièces d'esterlines

$54 \frac{1}{2} 2$ de rubans blancs

21 livres de fils d'argent

22 pièces de crées brodées

40 douzaines de papier de couleur de los chices

35 douzaines de bas de Bruxelles

93 livres de fils

10 écheveaux de fils primo

3 douzaines de escobilla de cabeza

Dans un autre fardeau :

18 pièces de rouens

9 pièces de rouens blancarts

7 pièces de rouens floretes

Chemises de Rouen, chemises de naval, mélinges, pièces de cotonies, pièces de morlaix, pointes de Paris, coûteaux de Palerme 


\section{RÉSUMÉ}

L'arrivée massive de métaux précieux américains en Andalousie dans les années 1570-1640 a été l'occasion pour les marchands de Saint-Malo et de Vitré de s'imposer sur les marchés espagnols, concurrençant ainsi les Espagnols qui s'étaient établis de longue date dans les ports français et, audelà dans le reste de l'Europe. Grâce à organisation basée sur la proximité des zones de production toilière, sur un commerce au comptant et la possession de navires armés, et dotés d'une vision beaucoup plus rationnelle des échanges, ils ont réussi à s'imposer comme d'authentiques acteurs de la première mondialisation, s'appuyant aussi bien sur une tradition de commerce de cabotage que sur des voyages d'exploration au long cours.

\section{ABSTRACT}

The mass arrival of American precious metals in Andalusia between 1570 and 1640 allowed Saint-Malo and Vitré traders to succeed in Spanish markets, competing with Spaniards well-established in French and other European ports. Thanks to an organisation based on neighbouring cloth production areas, cash payments and armed vessels, and with a better rational vision of trade, they were able to become genuine actors of the first globalisation, relying on short-sea trade tradition and long-distance exploration voyages. 